\title{
Correction to: Minimally Invasive Treatment for Benign Prostatic Hyperplasia: Economic Evaluation from a Standardized Hospital Case Costing System
}

\author{
Andrew D. Brown ${ }^{1} \cdot$ Steffan F. Stella ${ }^{2} \cdot$ Martin E. Simons ${ }^{1}$
}

Published online: 2 January 2019

(C) Springer Science+Business Media, LLC, part of Springer Nature and the Cardiovascular and Interventional Radiological Society of Europe (CIRSE) 2019

\section{Correction to: Cardiovasc Intervent Radiol \\ https://doi.org/10.1007/s00270-018-2132-8}

The original version of this article published previously contained an error. In Table 1, the mean total cost of PVP and TURP was transcribed incorrectly. The corrected Table 1 appears below:

The original article can be found online at https://doi.org/10.1007/ s00270-018-2132-8.

Andrew D. Brown

andrew.brown@uhn.ca

1 Division of Vascular and Interventional Radiology,

Department of Medical Imaging, Toronto General Hospital,

University Health Network, University of Toronto, 585

University Ave, Toronto, ON M5G 2N2, Canada

2 Division of Vascular and Interventional Radiology,

Department of Radiology, Hamilton Health Sciences,

McMaster University Medical Centre, McMaster University,

Hamilton, ON, Canada 
Table 1 Patient characteristics and overall costs for PAE, PVP and TURP

\begin{tabular}{lllll}
\hline & PAE & PVP & TURP & $P$ value \\
\hline $\begin{array}{l}\text { Age (years) } \\
\text { Mean (SD) }\end{array}$ & $70.77(8.72)$ & $73.66(7.14)$ & $71.43(8.37)$ & 0.366 \\
$\begin{array}{l}\text { Length of stay (days) } \\
\text { Mean (SD) }\end{array}$ & $1(0)$ & $1.55(0.73)$ & $1.63(1.47)$ & 0.076 \\
$\begin{array}{l}\text { Direct costs (\$) } \\
\text { Mean (SD) }\end{array}$ & $3143(1318)$ & $3573(966)$ & $3381(1380)$ & 0.478 \\
IQR & 1682 & 1424 & 1291 & \\
Minimum-maximum & $1252-6315$ & $1942-5327$ & $1147-14182$ & \\
Indirect costs (\$) & & & $1652(692)$ & \\
Mean (SD) & $677(406)$ & $2146(563)$ & 785 & \\
IQR & 391 & 868 & $577-5978$ & \\
Minimum-maximum & $381-1399$ & $1211-3227$ & & \\
Total cost $\$$ ) & & & $5034(1997)$ & 1917 \\
Mean (SD) & $3821(1582)$ & $5719(1515)$ & $1849-20160$ & \\
IQR & 1828 & 2248 & $3153-8554$ & \\
Minimum-maximum & $1665-7440$ & &
\end{tabular}

$I Q R$ interquartile range 